\title{
Intermédialités
}

Histoire et théorie des arts, des lettres et des techniques

Intermediality

History and Theory of the Arts, Literature and Technologies

\section{Optique ou haptique : le rythme dans les études sur l'art au début du $20^{\mathrm{e}}$ siècle}

\section{Georg Vasold}

Numéro 16, automne 2010

rythmer

rhythmize

URI : https://id.erudit.org/iderudit/1001955ar

DOI : https://doi.org/10.7202/1001955ar

Aller au sommaire du numéro

Éditeur(s)

Revue intermédialités (Presses de l’Université de Montréal)

ISSN

1705-8546 (imprimé)

1920-3136 (numérique)

Découvrir la revue

Citer cet article

Vasold, G. (2010). Optique ou haptique : le rythme dans les études sur l'art au début du $20^{\mathrm{e}}$ siècle. Intermédialités / Intermediality, (16), 35-55.

https://doi.org/10.7202/1001955ar
Résumé de l'article

Cet article traite du " rythme », une notion abondamment discutée lors de l'émergence d'une littérature académique en histoire de l'art. Malgré l'utilisation qu'en faisaient déjà Karl Schnaase et Franz Kugler, ce n'est qu'après 1900 que le terme deviendra un principe de base sous l'influence d'August Schmarsow, à Leipzig, et d'Alois Riegl, à Vienne. Ces derniers reconnaissaient l'importance et le potentiel d'une telle notion, malgré leurs travaux divergents. Pour August Schmarsow, le rythme était le moyen de concevoir la relation de l'esthétique et de l'expérience physique : traverser une architecture, un mouvement rythmique donc, décrivait un mode de perception élémentaire. En revanche, pour Riegl, le rythme était une question de vision : en explorant l'esthétique de l'Antiquité tardive (qui anticipait, selon lui, le modernisme), il observait davantage le rythme dans les rapports dynamiques entre le blanc et le noir, une idée qui deviendra pertinente pour la théorie cinématographique des années 1920. 


\title{
Optique ou haptique: \\ le rythme dans les études sur l'art au début du $20^{\mathrm{e}}$ siècle
}

\author{
Georg Vasold
}

E n août 1897 paraît dans Der Kunstwart, revue influencée par les mouvements réformateurs de l'époque, un article du jeune Oskar Bie, alors enseignant en histoire de l'art à la Technische Hochschule de Berlin et rédacteur en chef de l'influente Neue Deutsche Rundschau. Bie se penche sur un phénomène de son temps qui, comme il l'écrit lui-même, «ne semble pas insignifiant pour l'art ${ }^{1} »$. Il s'agirait d'une manifestation urbaine largement répandue qui «se prête à des considérations esthétiques [et] deviendra prochainement un facteur culturel² ».

Oskar Bie parle ici du cyclisme et son article pimenté d'ironie traite de l'esthétique de la bicyclette. Une citation plus détaillée du texte s'impose, car elle concentre tous les aspects pertinents au thème que nous voulons traiter :

Le deux roues est la seule chose qui ne puisse pas rester immobile dans la rue. Il tombe à l'arrêt, se relève dans la course. [...] C'est dans ce mouvement perpétuel forcé $[\ldots]$ qu'il trouve son âme. La simple maîtrise des difficultés fait que la bicyclette devient une partie de l'homme, elle met des ailes sur ses pieds et perd son caractère de machine. Tout débutant passe par le moment où il ressent, pour la première fois, la bicyclette qui se trouve sous lui comme faisant partie de lui-même: une onde légère se met tout à coup à parcourir son corps, il ne sent plus le fardeau de la machine. [...] Il se sent élastique, ailé, libre, et une sensation intense circule dans son sang. Ce sont là les symptômes donnés à l'homme dont les buts sont précis et la sensation nouvelle, cet homme qui doit prendre la relève du type scientifique, sceptique et décadent ${ }^{3}$.

1. Oskar Bie, «Fahrrad-Ästhetik», Der Kunstwart. Rundschau über Dichtung, Theater, Musik und bildende Kunst, ${ }^{\circ}{ }^{10}$, cahier $n^{\circ} 22,1897$, p. 339-340.

2. Ibid.

3. Ibid. 
Après cette introduction, qui laisse entrevoir que le sport n'est pas le seul sujet de l'article, Bie aborde l'art sans aucune transition :

La tendance formaliste de la vie artistique moderne a quelque chose de sain, si l'on entend par retour à la santé le retour à des facteurs de mouvement originels et simples. [...] Lorsque l'on puise ainsi à d'anciennes sources de création, comme c'est le cas aujourd'hui dans les premières lignes du combat de l'art, la sentimentalité décadente recule devant la poussée de la nature originelle et devant la joie du rythme pur. [...] Le rythme et l'étendue de l'horizon sont les tout premiers signes du formalisme renaissant. Celui-ci est la réaction à la dissymétrie exagérée du réalisme de la Bohème et à l'intimité exagérée de la décadence. Le mouvement du cycliste est la rythmiquet.

Oscar Bie dirige la Neue Deutsche Rundschau pendant 28 ans, de 1894 à 1922, et l'on peut supposer qu'aucune innovation intellectuelle ou tendance artistique en vigueur dans l'Allemagne wilhelminienne, et plus tard pendant la République de Weimar, ne lui a échappé. Il peut paraître étonnant au premier abord que, déjà en 1897, et avant Georg Simmel, il se soit interrogé sur la manière de faire face au stress de la grande ville et qu'il ait mis le rythme au cœur de ses propos afin d'expliquer la modernité ou, plus exactement, l'art moderne. Il suffit, pour comprendre la raison qui le pousse à accorder une telle importance au concept de rythme, de rappeler que Bie était historien de l'art. Or, autour de 1900, le rythme était effectivement l'un des termes les plus discutés en histoire de l'art, alors en émergence. On peut affirmer avec une certaine justesse que le rythme était même considéré comme un concept dominant de cette discipline, un concept qui paraissait tout bonnement indispensable aux chercheurs sur l'art. Quiconque passe la question du rythme au peigne fin dans les écrits des «vieux maîtres ${ }^{5}$ » spécialistes d'art moderne s'apercevra en effet que celle-ci était omniprésente. Presque tous les historiens de l'art dignes de ce nom en ont fait l'objet de leurs travaux: aussi bien Georg Dehio qu'Alois Riegl, Heinrich Wölflin, August Schmarsow, Wilhelm Pinder, Julius Meier-Graefe, Dagobert Frey ou Erwin Panofsky - pour ne mentionner que les plus connus.

Au vu d'un tel contexte, il est cependant aisé de reconnaître que l'usage de ce concept n'était pas du tout uniformisé au début du $20^{\mathrm{e}}$ siècle et que le mot «rythme» avait au contraire des significations très différentes les unes des autres et qu'il embrassait des phénomènes divers. Afin de parvenir à une compréhension

4. Ibid.

5. Heinrich Dilly (dir.), Altmeister moderner Kunstgeschichte, Berlin, Dietrich Reimer, 1990. 
historique de ce terme, il convient tout d'abord de démêler cet ensemble composé de multiples strates de signification. Le présent article entend contribuer à ce travail préliminaire en indiquant les raisons pour lesquelles ce concept a joui d'une aussi grande popularité auprès des historiens de l'art. Pour traiter cette question à l'aide d'un cas concret, je me concentrerai sur la querelle académique entre Alois Riegl et August Schmarsow - deux auteurs qui me paraissent exemplifier de manière paradigmatique l'usage hétérogène de ce concept.

\section{I.}

Les recherches sur le rythme connaissent présentement une petite renaissance: elles sont pour ainsi dire à la mode. Pour chaque année ou presque de la dernière décennie, au moins une monographie importante ou un article exhaustif ont paru sur le sujet. Chose curieuse, on constate que l'histoire de l'art occupe une place moindre dans ces publications. Et lorsque les arts visuels sont tout de même mentionnés dans les écrits dont nous disposons jusqu'ici, il s’agit presque exclusivement de l'œuvre de Paul Klee, pour qui le rythme constitue en effet une préoccupation centrale. En témoignent non seulement les nombreux tableaux dans lesquels il cherche à visualiser des structures musicales, mais aussi ses cours magistraux au Bauhaus où il expose aux étudiants la pertinence artistique des formes rythmiques ${ }^{6}$ (Fig. 1).

Klee n'est pourtant pas le seul peintre désireux de saisir l'essence du rythme. Il suffit ici de mentionner Piet Mondrian, Theo van Doesburg et plus particulièrement les futuristes italiens pour rendre tangible l'importance de ce concept dès les années 1910. Les pulsations de la ville comptent désormais parmi les topoi récurrents des manifestes futuristes et ce n'est donc pas étonnant que «Ritmo », un titre de tableau fréquemment repris, soit aussi bien utilisé par Carlo Carrà que Giacomo Balla.

Si l'on quitte la pratique artistique pour se tourner vers la recherche sur l'art, force est de constater que l'exploration intensive du rythme dans les arts visuels est accompagnée, voire préparée sur le plan théorique par les historiens de l'art. Le rythme revient en effet constamment dans les descriptions de phénomènes artistiques et ce, des dizaines d'années avant même que Klee et les futuristes ne fassent leur apparition. Ce n'est pas très surprenant, puisque après tout, le terme

6. Christiane Dessauer-Reiners, Das Rhythmische bei Paul Klee. Eine Studie zum genetischen Bildverfahren, Worms, Wernersche Verlagsgesellschaft, 1996; Toni Stooss et Tina Teufel (dir.), Paul Klee: Melodie/Rhythmus/Tanz, Weitra, Museum der Moderne Salzburg et Bibliothek der Provinz, 2008. 


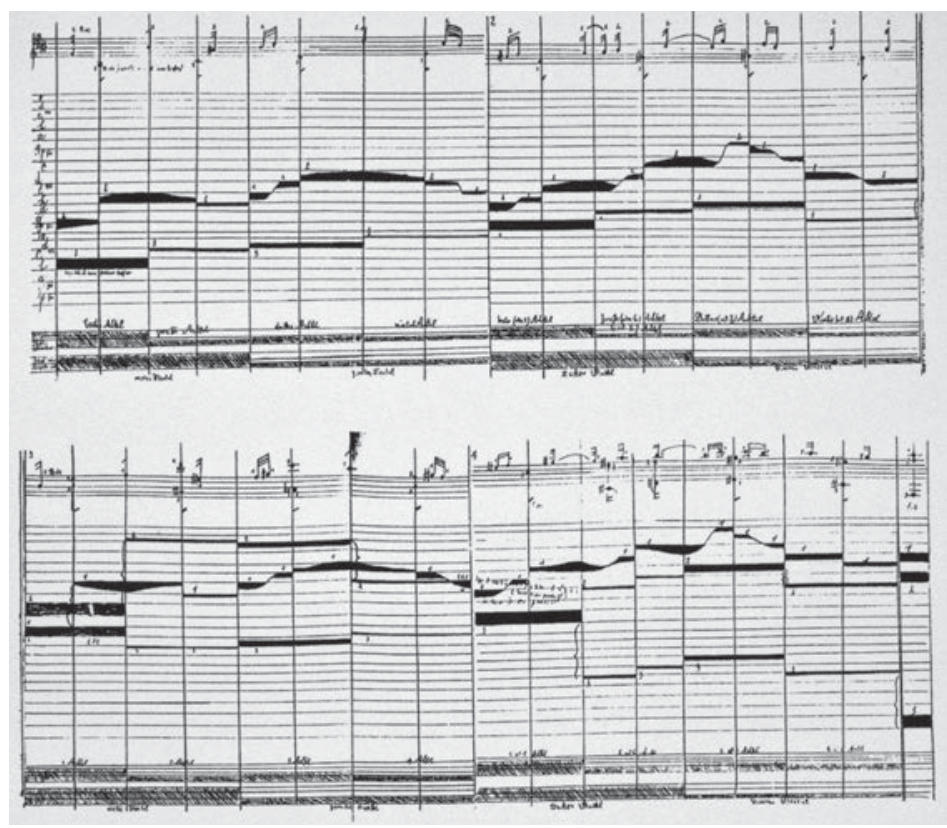

Fig. 1: Paul Klee, exemple pédagogique pour représenter les rythmes musicaux (maassrhythmische Darstellung), supplément au cours magistral du 16. o1. 1922 au Staatliches Bauhaus Weimar, Zentrum Paul Klee Bern (C) VBK, Wien 2010).

est en usage depuis l'Antiquité7. Sa particularité réside cependant dans le fait qu'il était presque toujours utilisé dans le contexte de la poétique, de la musique et de la danse jusqu'au $18^{\mathrm{e}}$ siècle. La situation change au $19^{\mathrm{e}}$ siècle et le concept est de plus en plus mobilisé pour décrire les arts visuels et surtout l'architecture. C'est le cas par exemple chez Karl Schnaase, mais encore davantage chez Franz Kugler, qui met notamment en relief deux aspects du rythme pertinents pour le développement ultérieur du concept. Il souligne tout d'abord l'aspect de la proportionnalité. Pour lui, le rythme réside dans les relations des composantes spatiales les unes avec les autres, et c'est d'ailleurs la raison pour laquelle il ne trouve presque aucun avantage aux prolongations du chœur : «L'allongement du chœur dérange [...] la relation rythmique de l'édifice ${ }^{8}$. Le second aspect mentionné par Kugler est encore plus important. Il est convaincu que le rythme

7. Wilhelm Seidel, «Rhythmus», dans Karlheinz Barck et al. (dir.), Ästhetische Grundbegriffe. Historisches Wörterbuch in sieben Bänden, vol. 5, Stuttgart et Weimar, J. B. Metzler, 2003, p. 291-314.

8. Franz Kugler, Geschichte der Baukunst, vol. 2, Stuttgart, Ebner und Seubert, 1858, p. $3^{85}$. 
entretient en tout temps une relation causale avec le mouvement. Ce constat n'est pas nouveau non plus, puisque les auteurs de l'Antiquité faisaient dériver

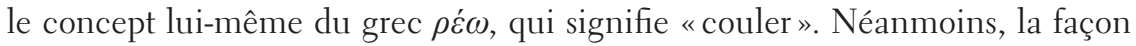
particulière dont Kugler parle du mouvement dans ses écrits sur l'architecture a un impact durable. Dans le troisième tome de son livre Geschichte der Baukunst consacré à l'art gothique français, on peut lire les propos suivants :

Un arrangement entièrement artistique est essentiel afin que ressorte toute l'ampleur des multiples variations du mouvement rythmique élémentaire dans les édifices plus richement conçus: les nefs latérales sont positionnées [...] en une voie polygonale autour de l'extrémité du chœur intérieur et sont elles-mêmes entourées d'un chapelet d'absides polygonales. [...] [L'extrémité du chœur gothique donne] l'image de l'organisme spatial le plus pur dont le mouvement élémentaire [...] trouve un aboutissement travaillé jusque dans le plus petit détail, rayonnant [à partir de la nef centrale] dans les pièces basses latérales pour aller ici se perdre dans des jeux rythmiques répétés dans la couronne des absides?.

Kugler décrit ici un mouvement rythmique élémentaire qui guide notre regard - «l'œil traversant l'espace» - à l'intérieur de l'édifice ${ }^{10}$. En lisant le livre de Kugler, on se tient dans l'église et on résonne d'emblée avec l'édifice grâce à l'uniformité de la structure des travées de voûte, puis on est propulsé par l'architecture elle-même, de niche en niche, dans un mouvement en avant autour du chœur, pour être finalement de nouveau libéré du rythme. S'accomplit ici, après que le chœur a été visité visuellement, pour ainsi dire, «la clôture rythmique du mouvement ${ }^{11} »$.

Force est de constater que les recherches sur la musique ne sont quasiment pas prises en considération dans la plupart des écrits sur l'histoire de l'architecture au $19^{\mathrm{e}}$ siècle $^{12}$. Ceci est d'autant plus surprenant qu'au moment précis où Kugler et Schnaase présentent leurs travaux, le rythme occupe une place prépondérante dans les recherches sur la musique. C'est le cas autour de 1845 à Vienne, où Eduard Hanslick déclare que le rythme constitue tout simplement l'essence même de la musique dans son étude pionnière Vom Musikalisch-Schönen.

9. Ibid., vol. 3,1859 , p. 11 et 23 .

10. Ibid., p. 23.

11. Ibid., p. 11.

12. Hans Hermann Russack, Der Begriff des Rhythmus bei den deutschen Kunsthistorikern des 19. Jahrhunderts, Weida (Thuringe), Thomas und Hubert, 1910, p. 19. 
Comme il l'écrit dans le chapitre trois, «l'élément originel de la musique est la sonorité et son essence, le rythme ${ }^{13} »$.

Au même moment à Leipzig, Thomaskantor Moritz Hauptmann va encore plus loin que Hanslick dans sa caractérisation du rythme. Pour lui également, sa signification ne fait aucun doute, mais il le définit en même temps par la négative, en l'opposant au mètre. C'est ici que l'aspect du mouvement joue de nouveau un rôle. «Nous nous proposons d'appeler mètre la mesure constante sur laquelle se base la mesure du temps et rythme, la nature du mouvement dans cette mesure ${ }^{14}$.» Ainsi, chez Hauptmann, l'écoutant est guidé de mesure en mesure dans le rythme de la mélodie à la manière de Kugler, pour qui l’observateur ou, mieux, le regard, est guidé rythmiquement à travers l'espace construit.

Toutes les idées développées au milieu du siècle pour caractériser le rythme et qui sont brièvement exposées ici réapparaissent dans les écrits de la génération suivante, chez Heinrich Wölfflin ou Georg Dehio par exemple. C'est toutefois pour August Schmarsow et Alois Riegl qu'elles ont le plus d'influence.

Si Schmarsow occupe une place très importante dans le cadre des recherches sur le rythme en histoire de l'art, c'est principalement parce qu'il consacre trois décennies à cet objet d'étude. Après le discours qu'il prononce à l'occasion de son entrée en fonction en tant que professeur à l'Université de Leipzig en 1893, il en fait le sujet de nombreuses communications et n'a de cesse de souligner qu'il constitue une catégorie absolument centrale de l'art. Le rythme est pour lui un «principe de configuration [Gestaltungsprinzip] ${ }^{15}$ » qu'il définit d'ailleurs plus précisément en le rattachant à la troisième dimension. Alors qu'il associe la symétrie à la largeur et la proportion à la hauteur, Schmarsow définit le rythme par la longueur ${ }^{16}$.

13. Eduard Hanslick, Vom Musikalisch-Schönen. Ein Beitrag zur Revision der Ästhetik der Tonkunst, Leipzig, R. Weigel, 1854. La présente citation est tirée d'une réédition: Darmstadt, Wissenschaftliche Buchgesellschaft, 1991, p. 32.

14. Moritz Hauptmann, Die Natur der Harmonik und Metrik, Leipzig, Breitkopf und Härtel, 1853, p. 223. La présente citation est tirée de Seidel, 2003, p. 307.

15. August Schmarsow, Grundbegriffe der Kunstwissenschaft. Am Übergang vom Altertum zum Mittelalter, Leipzig, B. G. Teubner, 1905. La présente citation est tirée de la nouvelle édition: Berlin, Gebrüder Mann, 1998, p. 85.

16. Schmarsow reprend ici la terminologie de Gottfried Semper qui parlait de direction au lieu de rythme: Gottfried Semper, Der Stil in den technischen und tektonischen Künsten, oder praktische Aesthetik. Ein Handbuch für Techniker, Künstler und Kunstfreunde, Münich, F. Bruckmann, 1860. Voir Eleftherios Ikonomoú, «Nachwort» dans Schmarsow, 1998, p. 355. 
Il serait déterminé par l'espace, ou, autrement dit, il serait un «critère de profondeur spatiale ${ }^{17} »$.

Le point critique, ou plutôt l'aspect décisif de l'ensemble de la théorie de Schmarsow réside dans la localisation du rythme dans le corps humain. Selon Schmarsow, l'architecture ne pourrait absolument pas être appréhendée autrement qu'« en faisant le tour de ses parties l'une après l'autre ${ }^{18} »$. Le rythme de la déambulation à travers l'espace se transforme ainsi en "critère d'ordonnancement des impressions ${ }^{19}{ }$. « Si la suite des complexes de stimuli s'accorde partout au rythme des mouvements corporels, l'expérience sera [...] vécue comme étant satisfaisante et bienfaisante ${ }^{20}$.»

Schmarsow affirme donc que l'édifice ne peut être vraiment réussi que si la déambulation à l'intérieur - comprise comme processus réel et physique - laisse un sentiment de résonance psychique chez le visiteur. Par-delà l'allusion au corps, il est facile de reconnaître ici la tentative d'enrichir l'histoire de l'art à l'aide de connaissances et de modes d'explication psychologiques. Les concepts choisis suffisent à le prouver, comme lorsqu'il est question de complexes de stimuli, d'appareil moteur, ou encore d'impulsions de la volonté et d'images mémorielles.

L'intérêt proclamé de Schmarsow pour les recherches en psychologie n'est pas surprenant. C'est en effet à cette même époque - c'est-à-dire pendant la dernière décennie du $19^{\mathrm{e}}$ siècle et la première décennie du $20^{\mathrm{e}}$ - que, non seulement l'histoire de l'art, mais aussi la physiologie de la perception et la psychologie émergente se découvrent un objet d'étude dans le rythme. L' American Journal of Psychology, dans lequel fut publiée une Bibliography of Rhythm contenant pas moins de 361 entrées, est peut-être le meilleur exemple à citer pour illustrer la popularité du rythme auprès des psychologues autour de $1900^{21}$. C'est à Leipzig,

17. August Schmarsow, «Über den Wert der Dimensionen im menschlichen Raumgebilde», Berichte über die Verhandlungen der Königlich-Sächsischen Gesellschaft der Wissenschaften in Leipzig. Philologisch-Historische Klasse, vol. 1, Leipzig, S. Hirzel, 1896. La présente citation est tirée de Beatrix Zug, Die Anthropologie des Raumes in der Architekturtheorie des frühen 20. Jahrhunderts, Tübingen, Ernst Wasmuth Verlag, 2006, p. 48.

18. Schmarsow, 1896. Cité d'après Zug, 2006, p. 46.

19. Ibid.

20. Ibid. Schmarsow se réfère aussi à Hermann Lotze, qui affirme que «l'homme se protège de la pléthore d'impressions sensorielles en adoptant une rythmisation intérieure involontaire». Voir Schmarsow, 1998, p. 85-86.

21. Christian A. Ruckmich, "Bibliography of Rhythm», The American Journal of Psychology, vol. 24, n 4, octobre 1913, p. 508-519. 
grand centre germanophone consacré à ce domaine de recherche, qu'officie Wilhelm Wundt, l'un des psychologues les plus renommés de son temps - un chercheur qui fait des incursions récurrentes en esthétique, ce qui l'amène à parler souvent du rythme. Schmarsow, lecteur enthousiaste de Wundt ${ }^{22}$, trouve dans les écrits de son collègue maintes inspirations et même plus que cela, puisque ses propres travaux peuvent bénéficier d'une attention de plus en plus accrue à l'ombre de la popularité dont jouit Wundt.

Si l'on se tourne à présent vers Vienne et Alois Riegl, on retient avant tout que Riegl a beaucoup moins écrit sur le rythme que ses collègues de Leipzig ${ }^{23}$. Riegl se sert du concept pour la première fois dans ses Stilfragen en 1893 et d'ailleurs seulement pour décrire des formes ornementales, même s'il lui accorde déjà le statut d'une «propriété artistique ${ }^{24}$ ». Ce point de vue est encore plus étoffé dans son étude Spätrömische Kunstindustrie, parue en 1901, où il déclare que le rythme est la caractéristique sine qua non de l'art antique, «le but ultime de toute activité artistique dans l'Antiquitée ${ }^{25}$ ». Pour soutenir une telle affirmation, il s'appuie sur deux œuvres de jeunesse de saint Augustin, De pulchro et apto, ainsi que De musica. Ce sont des écrits dans lesquels Augustin parle du rythme in extenso et s'interroge sur la perception de l'harmonie formelle et de la beauté dans l'art, ainsi que sur l'essence du laid. Riegl reprend ces réflexions formulées au $4^{\text {e }}$ siècle par le père de l'Église pour les développer en une théorie de l'intervalle, c'est-à-dire une théorie qui attribue à l'interstice une expression esthétique ayant une valeur propre. Alors que règne encore «la tendance à saisir la forme singulière ${ }^{26}$ » pendant l'Antiquité classique, un changement paradigmatique fondamental s'opère pendant l'Antiquité tardive. Pour la première fois dans l'histoire de l'art, il ne s'agit plus seulement de percevoir les motifs représentés - les figures se détachant d'un relief par exemple -, mais plutôt les parties qui se trouvent entre les figures (Fig. 2). Selon Riegl, l'Antiquité tardive est témoin de l'«émancipation de l'intervalle, ou l'élévation du fond neutre, informe,

22. Voir Zug, 2006, p. 49.

23. Michael Gubser entreprend de placer, pour la première fois, dans un contexte historique les réflexions de Riegl sur le rythme dans son essai intitulé «Rhythm in the Thoughts of Alois Riegl and his Contemporaries ", dans Peter Noever, Artur Rosenauer et Georg Vasold (dir.), Alois Riegl Revisited. Beiträge zu Werk und Rezeption, Vienne, Verlag der Österreichischen Akademie der Wissenschaften, 2010, p. 89-99.

24. Alois Riegl, Stilfragen. Grundlegungen zu einer Geschichte der Ornamentik, Berlin, G. Siemens 1893, p. 3.

25. Alois Riegl, Spätrömische Kunstindustrie, Berlin, Gebrüder Mann, 1992, p. 395-396. 26. Ibid., p. $39^{8}$. 


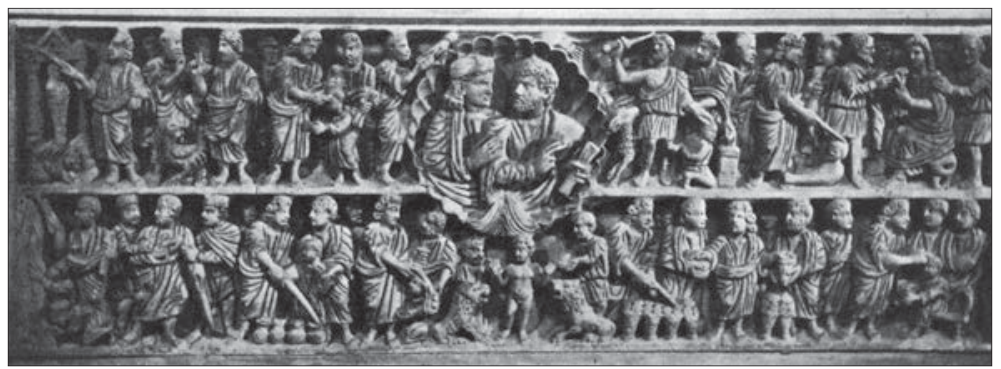

Fig. 2: Illustration tirée d'Alois Riegl, Spätrömische Kunstindustrie (Sarcophage, ca 5 e siècle, Rome, Museo del Laterano) (c) Fotosammlung des Instituts für Kunstgeschichte der Universität Wien.

en forme artistique latente ${ }^{27} »$. Cet entre-deux - le vide si l'on veut - n'est pas formé et semble sombre et indéterminé, tout particulièrement dans les reliefs sculptés en profondeur. C'est précisément cela, cependant, qui serait constitutif pour l'esthétique de l'Antiquité tardive, laquelle illustrerait «la justification de l'existence, voire la nécessité de l'informe ${ }^{28}$ ». Se référant à saint Augustin, Riegl affirme que cet entre-deux est «aussi nécessaire dans l'art que l'intervalle entre les paroles dans la langue [et] entre les sons en musique ${ }^{29}$ ». Pour Riegl, la caractéristique de l'art de l'Antiquité tardive qui détermine tout le reste réside dans la reconnaissance d'une égalité de valeur entre les parties formées et non formées, d'où la manifestation de cet art, en principe, sous la forme d'une coexistence rythmique entre les formes négatives et positives, ainsi que les endroits clairs et sombres. Riegl en tire deux conclusions: premièrement, comme nous pouvons le lire, le rythme est «nécessairement lié à la surface. Un rythme se compose d'éléments placés côte à côte ou les uns sur les autres, mais pas les uns derrière les autres ${ }^{30}$. L La deuxième conclusion concerne la forme spécifique de la perception. Pour pouvoir reconnaître le rythme en tant que tel, c'est-à-dire pour voir l'alternance entre la lumière et l'ombre, le noir et le blanc, l'observateur doit contempler l'objet à distance. C'est pour cette raison que les œuvres d'art de l'époque romaine tardive ne doivent pas être contemplées de près, mais seulement de loin, «en vue panoramique ${ }^{31}$ ».

À Leipzig, on réagit avec stupeur. Schmarsow apprend certes avec bonhomie que Riegl connaît désormais lui aussi la signification de l'art de l'Antiquité

$$
\begin{aligned}
& \text { 27. Ibid., p. } 390 . \\
& \text { 28. Ibid., p. } 399 . \\
& \text { 29. Ibid. } \\
& \text { 30. Ibid., p. } 389 . \\
& \text { 31. Ibid., p. } 399 .
\end{aligned}
$$


tardive en général, et du rythme en particulier. Mais il critique toutefois sévèrement l'interprétation répandue à Vienne. Dans sa réponse à Riegl, qu'il présente comme un «règlement de compte ${ }^{32}$ » et qui remplit tout un livre, il atteste des défaillances intellectuelles de Riegl: celui-ci ferait des «détours dangereux» et commettrait des «erreurs de pensée ${ }^{33}$ ». C'est notamment l'affirmation de Riegl selon laquelle le rythme serait impérativement lié à la surface que Schmarsow et ses disciples rejettent fermement ${ }^{34}$.

Il faut dire que l'atmosphère qui règne alors entre Leipzig et Vienne est quelque peu tendue. Riegl avait tenu des propos délibérément froids sur le livre de Schmarsow consacré au baroque ${ }^{35}$. En 1904, Max Dvořák rédige un commentaire dévastateur sur l'étude de Schmarsow sur la peinture du HautRhin $^{36}$; et lorsque Grundbegriffe de Schmarsow est finalement publié en 1905, Franz Wickhoff s'en prend à lui de front:

Il est préjudiciable à l'ensemble de la science que la chaire en histoire de l'art au sein d'une université telle que Leipzig soit occupée par un homme complètement ignorant de la signification de la recherche historique et ne comprenant rien aux problèmes élémentaires de l'histoire ${ }^{37}$.

À la lecture de ce compte-rendu, on est forcé de s'interroger: d'où vient ce ton, cette agressivité? La réponse est peut-être simplement celle-ci: on peut y voir la manifestation d'intrigues propres au milieu universitaire et considérer la querelle comme un simple jeu académique, plus ou moins courant, dont les enjeux sont l'hégémonie universitaire et l'autorité absolue sur un sujet précis. Certains éléments vont en effet dans ce sens. Les recherches sur l'Antiquité tardive deviennent un sujet à la mode dans les années 1890 et Vienne, leur

32. Schmarsow, 1998, p. VI.

33. Ibid., p. V.

34. Voir Willi Drost, Die Lehre vom Rhythmus in der heutigen Ästhetik der bildenden Künste, Leipzig et Gautzsch, Merkur, 1919, p. 24.

35. Alois Riegl, Die Entstehung der Barockkunst in Rom [1908], Münich et Mittenwald, Mäander, 1977, p. 15-16. La critique de Riegl visait l'étude de Schmarsow intitulée Barock und Rokkoko. Eine kritische Auseinandersetzung über das Malerische in der Architektur, Leipzig, Hirzel, 1897.

36. Max Dvořák, «Rez. von August Schmarsow, Die oberrheinische Malerei und ihre Nachbarn um die Mitte des 15. Jahrhunderts», Kunstgeschichtliche Anzeigen, vol. 1, n ${ }^{\circ}$, 1904, P. 12-24.

37. Franz Wickhoff, Abhandlungen, Vorträge und Anzeigen, 2. Bd.: Die Schriften Franz Wickhoffs herausgegeben von Max Dvořák, Berlin, Meyer et Jessen, 1913, p. 370. 
centre $^{38}$. Schmarsow entend apparemment renverser la situation, et c'est la raison pour laquelle il prend tellement à cœur les recherches sur le rythme. En témoignent ses nombreuses publications sur le sujet tout au long de sa vie, principalement dans la revue de Max Dessoir intitulée Zeitschrift für Ästhetik und allgemeine Kunstwissenschaft. Il semble, en outre, qu'il ait l'intention de créer une sorte de centre du rythme avec des collègues de l'Université de Leipzig issus d'autres disciplines, et les thèses sur le rythme sont par conséquent approuvées plus fréquemment à partir de 1900. Mais ce n'est pas tout: en 1901, l'historien et théoricien de la musique Hugo Riemann est appelé à travailler à Leipzig. Cet événement est déterminant car Riemann est le coryphée même de la recherche sur le rythme en musique et sa réputation s'étoffe encore plus lors de la publication de son étude System der musikalischen Rhythmik und Metrik en $1903^{39}$.

À Vienne aussi - et ceci témoigne encore une fois de la compétition qui règne entre les deux universités - on traque le rythme sans relâche, en couvrant une large palette de disciplines et de méthodes. Déjà en 1891, l'architecte Ferdinand von Fellner-Feldegg de l'Österreichisches Museum für Kunst und Industrie - c'est-àdire l'institution même où travaille Riegl - prononce un cours magistral ouvert au public sur le thème « Rythme, symétrie et proportion dans la nature et l'art ${ }^{40}$ »; le médecin Theodor Billroth écrit plusieurs essais sur le rythme dans la musique peu de temps avant sa mort en $1894^{41}$, et Friedrich Jodl se penche sur le mouvement rythmique des yeux lors de la perception de formes spatiales en $18966^{42}$.

On reconnaît donc facilement que les études sur le rythme sont alors l'enjeu d'intérêts stratégiques dans la recherche. Mais il est aussi probable que l'enthousiasme pour ce sujet ait un autre motif plus profond, motif intimement lié à la réflexion contemporaine sur la modernité.

38. Voir Georg Vasold, «Der Blick in den tragischen Spiegel. Zur kunsthistorischen Erforschung der Spätantike in Wien um 1900», dans Gábor Klaniczay et Ernő Marosi (dir.), The Nineteenth-Century Process of «Musealization» in Hungary and Europe, Budapest, Collegium Budapest Workshop, Series nº 17, 2006, p. 91-112.

39. Voir Christine Lubkoll, «Rhythmus. Zum Konnex von Lebensphilosophie und ästhetischer Moderne um 1900 ", dans Christine Lubkoll (dir.), Das Imaginäre des Fin de siècle. Ein Symposion für Gerhard Neumann, Freiburg im Breisgau, Rombach, 2002, p. 83-110; Hans-Joachim Hinrichsen, «Musikalische Rhythmustheorien um 1900», dans Barbara Naumann (dir.), Rhythmus. Spuren eines Wechselspiels in Künsten und Wissenschaften, Würzburg, Königshausen und Neumann, 2005, p. 141-156.

40 Voir un court synopsis de l'exposé dans: Mittheilungen des k.k. Oesterreichischen Museums für Kunst und Industrie, tome 4, 1892-1893, p. 32-33.

41. Theodor Billroth, Wer ist musikalisch?, Berlin, Paetel, 1895.

42. Friedrich Jodl, Lehrbuch der Psychologie, Stuttgart, Cotta, 1896, p. 317-374. 
II.

Si le rythme est de toute évidence aussi prisé et contesté, c'est parce que le véritable enjeu du débat n'est rien de moins que la vie elle-même. Autour de 1900 , on associe en effet presque constamment le rythme à la vie pour des raisons évidentes. De nombreux textes de l'époque font référence au battement du cœur comme étant le rythme vital même. De telles idées entrent également dans les travaux en histoire de l'art. Schmarsow lui-même rattache la théorie du rythme à la démarche de l'homme ${ }^{43}$, et Heinrich Wölfflin lui emboîte le pas lorsqu'il écrit que «nous respirons avec régularité, nous marchons avec régularité, chaque activité continue s'accomplit dans une suite périodique ${ }^{44} »$.

C'est précisément cela qui intéresse un homme dont l'influence est déterminante pour la pensée de Riegl et qui met toute son énergie à cerner l'implication d'une telle activité rythmique continue et constante. Il s'agit de Karl Bücher, économiste à Leipzig (mais dans quelle autre ville? est-on tenté de se demander), qui analyse les processus de travail pendant des années. L'étude qui en résulte, Arbeit und Rhythmus (1897), connaît une telle popularité qu'elle est rééditée six fois jusqu'en $1924^{45}$. Bücher s'y demande, entre autres, quelle est la signification des activités rythmiques, des gestes à peu près constants accomplis pendant le travail, ou plus précisément quelles conséquences ceux-ci peuvent-ils avoir, à la fois pour les travailleurs et pour les produits fabriqués. Cette question est d'actualité dans la politique des affaires courantes à cause des bouleversements économiques qui ébranlent le $19^{\mathrm{e}}$ siècle. À partir des années 1880 en Allemagne justement, et ensuite un peu plus tard en Autriche, les conséquences de l'industrialisation font l'objet de débats houleux. Cette discussion intéresse aussi les historiens de l'art, surtout lorsque ceux-ci, tel Alois Riegl, traitent de questions relatives au dit art industriel ${ }^{46}$. Karl Bücher étudie ainsi l'impact des chants rythmant le travail sur la production d'art artisanal dans un chapitre consacré au sujet. Bücher observe par exemple qu'on chante beaucoup à la campagne, non

43. Voir Zug, 2006, p. 48.

44. Heinrich Wölfflin, Prolegomena zu einer Psychologie der Architektur, Münich, C. Wolf, 1886. La présente citation est tirée de la nouvelle édition Berlin, Gebrüder Mann, 1999, p. 21.

45. Karl Bücher, Arbeit und Rhythmus. Abhandlungen der philologisch-historischen Classe der königlichen sächsischen Gesellschaft der Wissenschaften, Leipzig, S. Hirzel, 1896. 46. Sur l'influence de Bücher sur Riegl, voir Georg Vasold, Alois Riegl und die Kunstgeschichte als Kulturgeschichte. Überlegungen zum Frühwerk des Wiener Gelehrten, Freiburg im Breisgau, Rombach, 2004, p. 120-134. 
parce que la vie y est plus agréable, mais parce que le rythme des chants permet de mieux structurer le travail. Il inclut des exemples de chansons entonnées pendant le tissage - une activité hautement rythmique en soi. Il n'est pas étonnant qu'Alois Riegl, pendant longtemps conservateur des tissus à l'Österreichisches Museum für Kunst und Industrie, soit devenu un lecteur enthousiaste de Karl Bücher. Et ceci d'autant moins que, dans son étude, Bücher cherche aussi à savoir si le rythme ne serait pas une sorte de force spéciale capable de générer des formes, une force qui en fin de compte produirait l'art et s'inscrirait par là dans une relation causale avec la vie.

Il est frappant de constater à quel point l'idée selon laquelle le rythme donnerait naissance à l'art et lui insufflerait la vie par la même occasion est fréquente en 1900. Herwarth Walden écrit en effet à ce sujet que «le mouvement structuré, le rythme, est la vie de l'œuvre d'art ${ }^{47} »$. Et le théoricien de la musique Hugo Riemann, déjà mentionné, consigne sans équivoque que «le rythme n’est pas un principe d'organisation imaginaire, mais plutôt le contenu musical, la vie à l'intérieur de segments temporels délimités ${ }^{48}$ ». Mais ce n'est pas tout, car le rythme dans sa vivacité est également décrit comme un phénomène éminemment moderne et donc éminemment populaire, c'est-à-dire comme un phénomène lié au mode de vie d'une large couche de population. Le texte d'Oscar Bie cité plus haut (celui-ci a d'ailleurs étudié à Leipzig) représente le prélude à d'autres publications dans lesquelles l'auteur analyse les activités rythmiques décisives pour l'époque moderne et qui prennent ancrage dans le corps. Suivant le principe que "toutes sortes d'activités sont rythmiques», il examine, par exemple, le contenu rythmique de loisirs populaires tels que le tennis ou la danse ${ }^{49}$ - activité dont Siegfried Kracauer dit comme chacun sait qu'on «s'y adonne aujourd'hui avec un dévouement [...] jamais égalé dans les époques

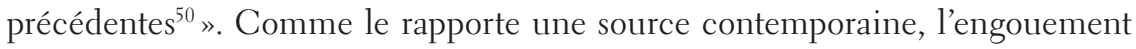
généralisé pour la danse vient du fait que le rythme rend possible la création d'un sentiment de bonheur collectif. «Les mouvements rythmiques du corps humain dans l'espace [peuvent] entraîner d'autres individus dans des vibrations

47. Herwarth Walden, Die neue Malerei, Berlin, Verlag Der Sturm, 1919. Cité d'après Dessauer-Reiners, 1996, p. 14, note 1.

48. Cité d'après ibid., p. 39.

49. Oskar Bie, Der Tanz, Berlin, J. Bard, 1919.

50. Siegfried Krakauer, Das Ornament der Masse, Frankfurt am Main, Suhrkamp, 1977, p. 40. 
égales ou comparables et, ce faisant, les [mettre] dans un état d'ivresse égal ou comparable ${ }^{51}$.»

Il est surprenant de constater que l'emprise du rythme sur la vie de l'homme moderne, aussi bien dans ses moments de travail que dans ses loisirs, se retrouve également chez Riegl. On a étrangement l'impression qu'il écrit en fait aussi sur des phénomènes contemporains. Qu'il s'interroge sur les ramifications de l'industrialisation en Autriche-Hongrie dans son analyse de l'art populaire, ou qu'il se déclare en faveur de l'art issu d'une communauté égalitaire - la république des sept provinces unies - dans Holländisches Gruppenporträt (1902), il établit toujours un lien clair avec la société ou la politique, et le plus souvent avec la société contemporaine. C'est dans Spätrömische Kunstindustrie que cette impression domine tout particulièrement ${ }^{52}$. Dès que l'on commence à placer les propos de Riegl sur l'Antiquité tardive dans un contexte contemporain, il devient évident que la réflexion porte ici - obliquement ou en reflétant le contexte historique - sur la situation culturelle et politique de l'Europe des années 1900. En vérité, les aspects centraux de son livre se laissent transposer sans difficulté dans l'époque que constitue le tournant du siècle: il s'agit avant tout d'un questionnement, remis au cœur des débats depuis Jacob Burckhardt, sur les circonstances, y compris temporelles, qui déterminent la chute d'un grand empire, ainsi que sur les phénomènes causés par la défaite politique et sur le sens profond de la décadence. Le phénomène de la migration occupe aussi le premier plan, autrement dit, on s'interroge sur la manifestation extérieure d'influences culturelles, ce qui amène par conséquent à considérer le problème des continuités historiques. Des réflexions fondamentales sur l'hégémonie culturelle ainsi que sur l'héritage culturel, telles qu'elles se manifestent notamment dans l'état habsbourgeois multiethnique, résonnent dans Spätrömische Kunstindustrie. Et finalement, il s'agit dans tout cela d'un thème-clé de la modernité en tant que telle, à savoir la relation entre une haute culture dominante et une myriade de cultures populaires souvent dissidentes.

En lisant Riegl de cette manière, on n'est plus vraiment surpris de trouver dans Spätrömische Kunstindustrie un concept politique central à la fin du

51. Georg Fuchs, Der Tanz, Stuttgart, Strecker und Schröder, 1906, p. 13, cité d'après Erika Fischer-Lichte, Ästhetik des Performativen, Frankfurt am Main, Suhrkamp 2004, p. 97.

52. Margaret Olin, «Alois Riegl: The Late Roman Empire in the Late Habsburg Empire», dans Ritchie Robertson et Edward Timms (dir.), The Habsburg Legacy: National Identity in Historical Perspective, Austrian Studies, vol. 5, Edinburgh, Edinburgh University Press, 1994, p. 107-120. 
$19^{\mathrm{e}}$ siècle. Ce concept, qui marque peut-être le plus le paysage politique de l'Autriche et de Vienne sous l'influence de Karl Lueger en particulier, est le concept de masses ${ }^{53}$. Ce terme semble venu de nulle part dans Spätrömische Kunstindustrie, et Riegl s'en sert pour donner une définition plus précise du rythme. L'art de l'Antiquité tardive exhiberait dans sa composition essentiellement «rythmique » un «nivellement du fond et de la forme particulière», ce qui mènerait à une composition de masse (Massenkomposition), pour reprendre les mots de Riegl: «une manifestation inouïe au sein de l'art antique [qui] représente l'ébauche de la conception moderne du caractère collectif de la forme singulière en apparence ${ }^{54}$ ».

Pour Riegl, le dépassement de la forme singulière, l'abandon de la manifestation individuelle et sa dissolution dans le collectif sont modernes et inouïs dans leur modernité. Cela caractérise l'homme moderne et trouve sa préfiguration dans l'art de l'Antiquité tardive. Pour apprécier cette découverte à sa juste valeur, on doit rappeler que Riegl travaille sur son essai monumental Das holländischen Gruppenporträt pendant qu'il rédige Spätrömische Kunstindustrie (il y passe huit années). C'est un texte dans lequel il s'agit, entre autres, de savoir de quelle manière il est possible de représenter visuellement un groupe d'hommes sans négliger le caractère individuel de chacun. Il est évident que le même problème préoccupe Riegl pour ce qui est de l'Antiquité tardive. Selon Riegl, le regard à distance dissoudrait l'individu et il ne subsisterait que l'impression rythmique des masses en mouvement: «Plus la figure singulière est en mouvement, plus elle devient difficile à distinguer. Le sentiment de l'absolue nécessité de l'individualité diminue. Le principe de regroupement promeut cela ${ }^{55}$.»

Riegl entend visiblement comprendre comment les masses - le collectifpeuvent être représentées. On peut aisément trouver des réponses à ce questionnement à l'époque de la monarchie habsbourgeoise tardive, où des rassemblements de masse sont à l'ordre du jour pour des raisons politiques, et où la photographie semble être le médium le plus adéquat pour représenter les masses. Car on voit très clairement dans les photographies historiques en noir et blanc l'alternance justement décrite par Riegl entre les parties claires et sombres qui se combinent

53. Helmut König fait référence à Vienne comme centre européen d'une politique de masse dans Zivilisation und Leidenschaften. Die Masse im bürgerlichen Zeitalter, Reinbek bei Hamburg, Rowohlt, 1992, p. 192-199.

54. Riegl, 1992, p. 391.

55. Alois Riegl, Historische Grammatik der bildenden Künste, Graz et Cologne, H. Böhlau, 1966, p. 279. 


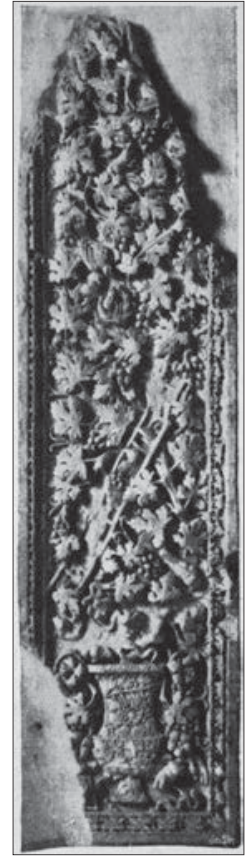

Fig. 3: Illustration tirée d'Alois Riegl, Spätrömische Kunstindustrie (Pilier en marbre, ca. $3^{\mathrm{e}}$ siècle, Rome, Museo del Laterano) (C) Fotosammlung des Instituts für Kunstgeschichte der Universität Wien. pour former un tout, un collectif, seulement dans la vue d'ensemble. Il en est pour les photos de masses humaines exactement comme pour un pilastre de marbre représenté dans Spätrömische Kunstindustrie, où il devient impossible pour l'observateur de différencier les sarments, feuilles et grappes parce que tout se confond dans un mouvement de croissance et d'enroulement du végétal (Fig. 3): dans les deux cas, leur caractère, soit leur vivacité et leur mobilité, ne se révèle que dans la vue d'ensemble (Fig. 4).

Ces réflexions sont révélatrices des différences entre Schmarsow et Riegl. Tout compte fait, ils parlent de choses complètement différentes dans leur interprétation du rythme. Tandis que Schmarsow analyse l'espace artistique puisqu'il estime que c'est seulement là - dans la troisième dimension - qu'une expérience du rythme est possible, Riegl nomme la planéité [Flächigkeit] comme caractéristique du rythme. Et alors que Schmarsow considère l'observateur comme un participant actif dans le processus artistique, Riegl insiste sur le fait que l'art - dans sa qualité rythmique justement - n'intéresse pas la perception corporelle et haptique, mais plutôt la perception optique et contemplative.

\section{III.}

Pour autant que ces deux chercheurs aient abordé l'histoire de l'art de manière complètement différente, leurs conceptions du rythme s'avèrent extrêmement influentes, chacune à sa façon.

Il n'est plus nécessaire aujourd'hui de souligner la multiplicité des chemins qui conduisent de Riegl au $20^{e}$ siècle. Ce n'est que récemment que l'importance des réflexions de celui-ci pour la théorie artistique de la modernité a été documentée dans le détail. Les écrits de Clement Greenberg en particulier semblent impensables sans le travail préliminaire de Riegl, comme en témoigne «Modernist Painting» (1960) de Greenberg - un texte stipulant, comme on le 


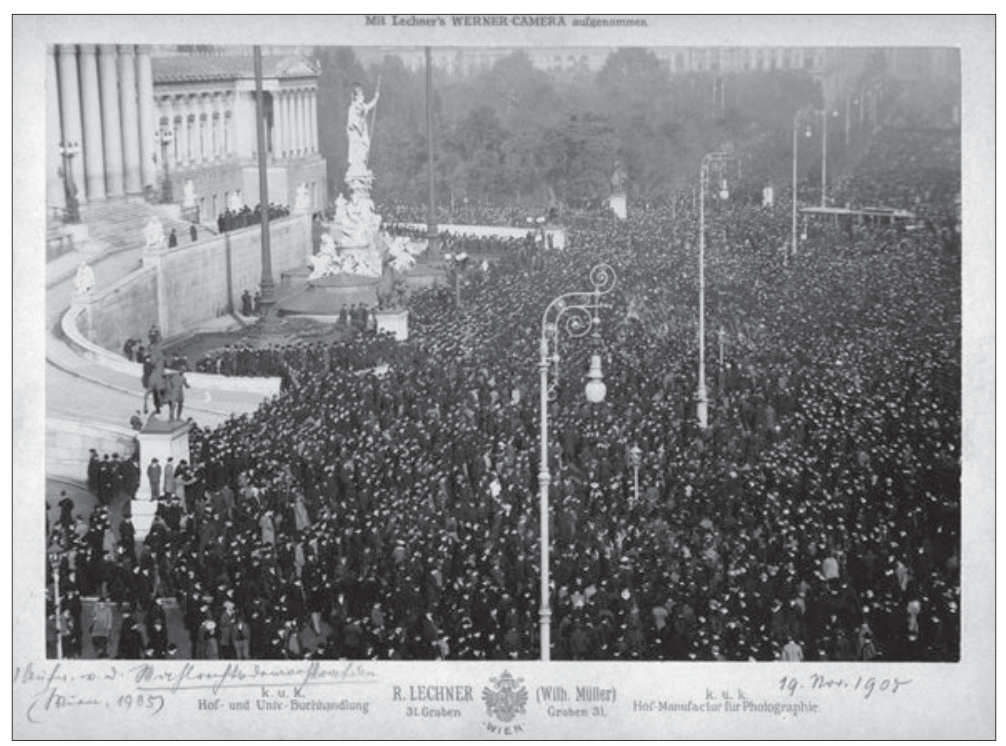

Fig. 4: Manifestation pour le droit de vote devant le Parlement de Vienne, le 19 novembre 1905. (C) Bildarchiv der Österreichischen Nationalbibliothek.

sait, que l'aplat est le paradigme par excellence de la peinture moderne ${ }^{56}$. Mais Riegl semble aussi avoir influencé d'autres disciplines. Lorsqu'il propage le penchant esthétique de l'Antiquité tardive pour l'alternance constante du clair et de l'obscur, ou qu'il définit l'observateur, non pas par le mouvement comme chez Schmarsow, mais plutôt par la contemplation immobile et à distance du mouvement rythmique entre le noir et le blanc, où ce qui est vu est strictement lié à la surface, la question se pose de savoir, d'une part, en quoi consiste véritablement la relation entre la photographie et la conception de la théorie de l'histoire de l'art au tournant du $20^{\mathrm{e}}$ siècle, et aussi s'il n'y a pas ici une sorte d'esthétique filmique avant la lettre.

De fait, Dziga Vertov pourrait avoir renvoyé à Riegl57, et il n'est pas le seul. Le pionnier du film abstrait Hans Richter le fait aussi, consciemment ou non.

56. Regine Prange, «Konjunkturen des Optischen. Riegls Grundbegriffe und die Kanonisierung der künstlerischen Moderne », dans Noever, Rosenauer et Vasold, 2010, p. $109-128$.

57. Vera Kropf a présenté l'exploration du rythme par Dziga Vertov dans un essai récent intitulé «Der Film als Rhythmus. Ansätze zur Untersuchung visueller Rhythmen am Beispiel von Dziga Vertovs Odinnadcatyj (SU 1928)", Maske und Kothurn, ${ }^{\circ}{ }_{55}$, cahier 3, 2009, p. 97-114. 


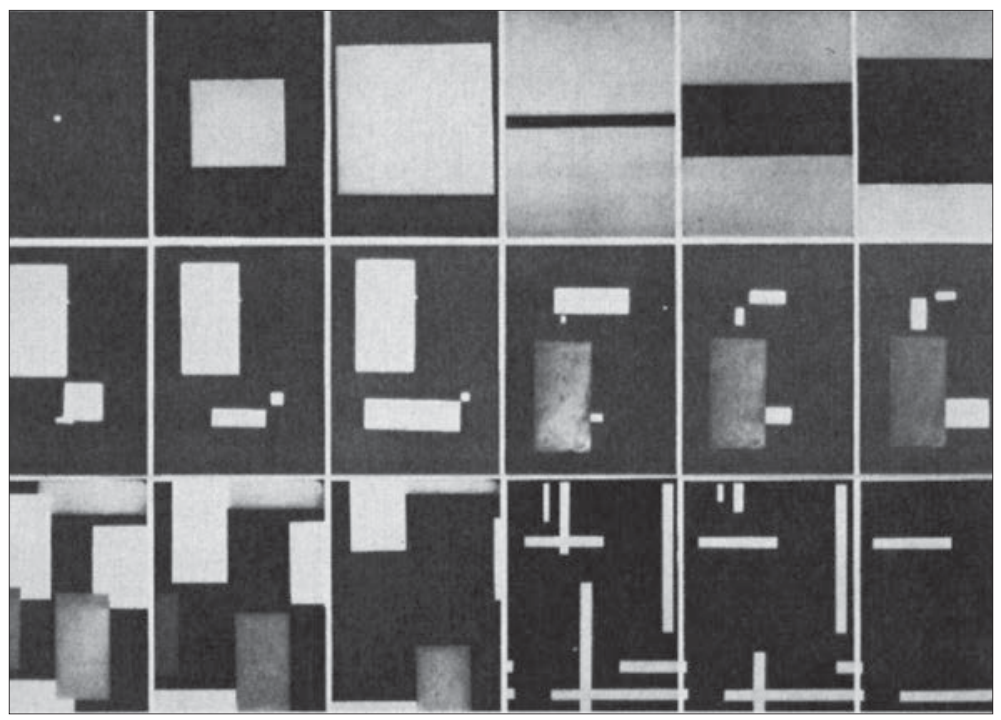

Fig. 5: Photogramme extrait de Rhythmus 21 de Hans Richters (1921).

Dans un entretien réalisé peu de temps avant sa mort en 1976, Richter souligne énergiquement la signification de l'interstice et de l'utilisation égale des formes positives et négatives ${ }^{58}$. Son film Rhythmus 21 (1921) fait défiler rythmiquement des surfaces noires et blanches pendant 3 min $5 \mathrm{~s}$, c'est-à-dire qu'il emploie une esthétique basée sur les relations entre le clair et l'obscur remontant sans doute à Riegl, comme on l'a supposé récemment ${ }^{59}$ (Fig. 5). Et de fait, dans Spätrömische Kunstindustrie, celui-ci se penche sur la signification esthétique de la fenêtre qui «[perce] un trou dans le mur, un rien sans consistance, semblable à l'ombre». Dans l'observation à distance se produirait ainsi une «alternance rythmique [...] de percées sombres et de surfaces murales claires ${ }^{60}$ ». Établie de cette manière, cette reconnaissance esthétique de l'interstice entraînerait la dissimulation de la relation entre le motif et le fond, phénomène qui serait très clairement visible dans les bronzes ajourés de l'Antiquité tardive. Sur ces broches et fibules, les parties claires et obscures seraient distribuées de manière complètement égale.

58. Hans Richter, Early Works, DVD, Paris, RE:VOIR-Video. Voir aussi sur le même sujet Hans Richter, Film ist Rhythmus, Berlin, Freunde der Kinemathek, 2003.

59. Robin Curtis et Marc Glöde, «Haptische Rhythmen: Visuelle Intervalle in der filmischen Wahrnehmung», dans Christa Brüstle, Nadia Ghattas, Clemens Risi et Sabine Schouten (dir.), Aus dem Takt. Rhythmus in Kunst, Kultur und Natur, Bielefeld, Transcript, 2005, p. 269-288.

6o. Riegl, 1992, p. 49-50. 


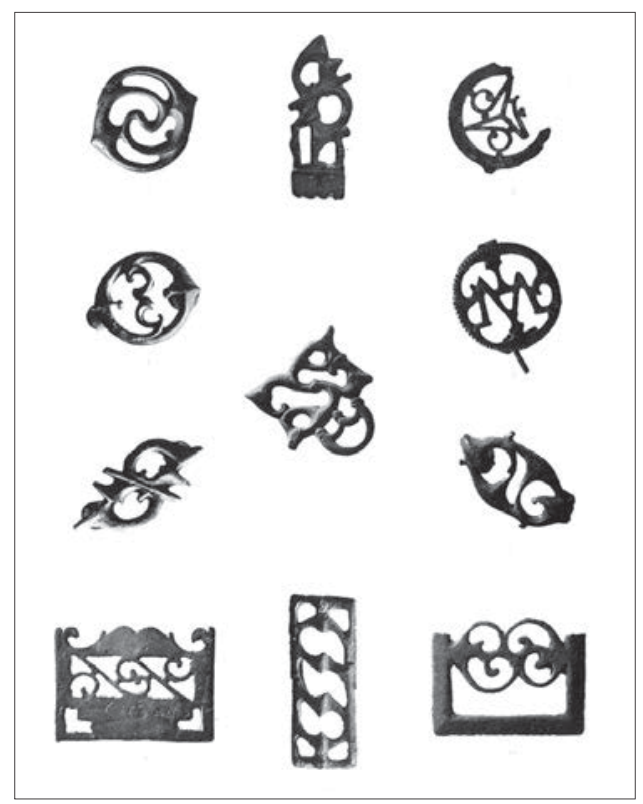

Fig. 6: Illustration tirée d'Alois Riegl, Spätrömische Kunstindustrie (Pièces de bronze découpées à l'emporte-pièce datant de l'époque romaine tardive) (C) Fotosammlung des Instituts für Kunstgeschichte der Universität Wien.

En somme, l'«agencement du clair et de l'obscur dans l'alternance ${ }^{61}{ }$ ne permet pas de savoir ce qui est plus important pour l'impression générale: les passages obscurs ou les percées claires (Fig. 6).

Cependant, il faut admettre que non seulement Riegl est en avance sur son temps à bien des égards, mais c'est aussi le cas des réflexions de Schmarsow, dont la pertinence ne sera cependant reconnue que beaucoup plus tard. Contrairement à ce que pense Wickhoff, il ne s'agit pas de paroles vides dans l'œuvre de Schmarsow, mais d'un premier exemple de ce qu'on nommera plus tard l'esthétique de la réception en histoire de l'art ${ }^{62}$. Il s'agit cependant d'une esthétique de la réception qui va au-delà de la pure observation. Ce que Schmarsow n'a de cesse de revendiquer pendant des décennies, c'est la signification du corps dans l'art. L'expérience de l'art, l'expérience vécue de l'architecture pour ainsi dire, gagne en ce sens une dimension anthropologique, comme le démontre Beatrix Zug de manière convaincante. L'individu agissant est reconnu

61. Ibid., p. 142.

62. Wolfgang Kemp (dir.), Der Betrachter ist im Bild. Kunstwissenschaft und Rezeptionsästhetik, Berlin, D. Reimer, 1992, p. 51-59. 
par Schmarsow comme «valeur dominante» dans le cadre de l'art visuel. Pour lui, l'être humain fait partie intégrante de l'«expérience vécue de l'art», un «principe [qui prend] sa source dans le sujet».

Chez Schmarsow, il ne s'agit donc pas seulement de reconnaître l'observateur comme un receveur. Bien plus, il développe, dans l'ombre des recherches sur le rythme, une théorie qui vise en dernier lieu à définir l'être humain dans son interaction avec l'art, à réclamer sa participation active au processus artistique, à le rendre tout simplement auteur.

C'est une position qui s'est de nouveau imposée avec force au centre des recherches des dernières années, dans lesquelles il est beaucoup question de performativité. Il revient aux recherches d'Erika Fischer-Lichte d'avoir au moins commencé à appréhender le rythme dans sa dimension historique. Elle indique à plusieurs reprises que la culture européenne autour de 1900 aurait effectué la transition d'une culture dominée par la textualité à une culture où domine la performativité, et que le discours sur le rythme joue un rôle crucial dans ce processus. Il est regrettable que l'on ne sache pas plus à quel point l'histoire de l'art y contribue. Car Schmarsow n'est en effet pas le seul à reconnaître le contenu artistique et performatif du rythme. Que l'on se souvienne encore une fois d'Oskar Bie qui, ayant rencontré le rythme en 1897, écrit presque exclusivement sur l'opéra, la danse, le théâtre et, par la suite, la musique.

La génération des historiens de l'art actifs autour de 1900 n'est pas la dernière à se pencher sur le rythme. En effet, les écrits de Riegl, Schmarsow ou Bie représentent seulement un prélude à un engouement pour ce sujet qui enfle jusqu'en 1925 environ. La raison se trouve évidemment aussi dans l'histoire de la discipline même dans laquelle on ressent encore la nécessité impérieuse de trouver une terminologie stable. Le rythme paraît remplir, pendant un temps, toutes les exigences associées à un concept authentique de l'histoire de l'art. On a vu qu'il n'est pas seulement valable pour l'analyse formelle, mais qu'il doit en plus être compris comme un principe culturel d'organisation global. Avec lui, on a trouvé un terme qui organise la forme de l'image et classe les données visuelles, tout en tenant compte aussi de la dimension temporelle, un terme qui renvoie au mode de production, qui permet de faire des implications politiques et sociales et qui s'ancre toujours dans le ressenti humain, c'est-à-dire dans le corps.

La disparition ultérieure du rythme de la discipline est liée à la marche triomphale de l'écriture formaliste de l'histoire de l'art telle que la pratique Wölfflin. Lorsque Heinrich Wölfflin publie son étude Kunstgeschichtliche Grundbegriffe en 1915, il donne à la discipline une direction très différente de celle que Riegl et Schmarsow ont empruntée. Wölfflin entraîne la recherche 
dans un domaine où le corps n'a pas de place et où ne se trouve aucun point de référence pour la compréhension de l'art moderne. Son livre, canonique pour la recherche germanophone et qui compte encore parmi les lectures obligatoires dans un grand nombre d'universités, présente dix concepts permettant de nommer et de comprendre les manifestations de l'art visuel, notamment celles de la Renaissance et du Baroque. Le rythme qui avait joué un rôle important dans ses premiers écrits n’en faisait plus partie.

Traduit de l'allemand par Hélène Sicard-Cowan 